\title{
Radiographic appearance and clinical implications of the presence of radix entomolaris and radix paramolaris
}

\author{
T.K. Różyło, M.J. Piskórz, I.K. Różyło-Kalinowska \\ Department of Dental and Maxillofacial Radiodiagnostics, Medical University in Lublin, Poland \\ [Received 27 March 2014; Accepted 28 May 2014]
}

Background: The variation in mandibular molars' anatomy is not as rare as it might appear to be. Depending on the location of the additional root found in a mandibular molar, it may be referred to as either radix entomolaris (RE) or radix paramolaris $(R P)$. The aim of the study was to present radiographic appearance of RE and RP and indicate the clinical implications of its presence.

Materials and methods: The material consisted of 3,000 panoramic and 300 periapical radiographs of first and second mandibular molars taken in the Department of Dental and Maxillofacial Radiodiagnostics of the Medical University in Lublin which were screened for RE and RP.

Results: Among panoramic and periapical radiographs only 14 cases of additional roots were found: 11 teeth with RE/RP in the Polish population and 3 in Taiwanese. Only 2 panoramic radiographs revealed the presence of RE and surprisingly the occurrence was bilateral.

Conclusions: Dentists should not be taken by surprise at finding an additional root in mandibular molars. They should be aware of the anatomical diversity of these teeth and know how to recognise possible extra roots during radiograph reading. (Folia Morphol 2014; 73, 4: 449-454)

Key words: anatomy variation, cone beam computed tomography, mandibular molars anatomy, radix entomolaris, radix paramolaris, radiological examination

\section{INTRODUCTION}

The variation in teeth anatomy is not as rare as it might appear to be. Commonly known as 2-rooted teeth, molars have also been found to occur with an additional root - either distolingual or mesiobuccal. It was in 1844 when Carabelli discovered an extra root in mandibular molars, subsequently referred to as radix entomolaris (RE). RE is located distolingually to the mesial root of a mandibular molar. However, this anatomic variation in mandibular molars was not the only one, as later on Bolk found an additional root which was located mesiobucally and named it radix paramolaris (RP) [12]. In the latest papers RE is also frequently referred to as distolingual root $[15,21]$.

So far, the theories concerning the origin of RE and RP have been postulated. According to the first one, $\mathrm{RE}$ and RP form as a result of external determinants during odontogenesis, whereas the second theory indicates that an atavistic gene may be responsible for the occurrence of RE and RP. The genetic theory is justified by the fact that reversions could be observed in the next generations of the trait [12].

Address for correspondence: M.J. Piskórz, DDS, Department of Dental and Maxillofacial Radiodiagnostics, Medical University in Lublin, ul. Karmelicka 7, 20-081 Lublin, Poland, e-mail: magdalena.piskorz@umlub.pl 
Table 1. Characteristics of the diagnosed supernumerary roots

\begin{tabular}{|c|c|c|c|c|c|c|c|c|}
\hline No. & Initials & Gender & Race & $\begin{array}{l}\text { Type of } \\
\text { radiograph }\end{array}$ & Tooth & Side & $\begin{array}{l}\text { Type of } \\
\text { radix }\end{array}$ & $\begin{array}{l}\text { Image of radix entomolaris } \\
\text { according to Wang's } \\
\text { classification }\end{array}$ \\
\hline 1. & M.A. & Female & Caucasian & Periapical & Second molar & Right & Entomolaris & Slightly overlapped \\
\hline 2. & M.B. & Male & Caucasian & Periapical & First molar & Left & Entomolaris & Moderately overlapped \\
\hline 3. & C.C.H. & Male & Asian & Periapical & First molar & Left & Entomolaris & Moderately overlapped \\
\hline 4. & S.C. & Female & Caucasian & Periapical & First molar & Left & Entomolaris & Severely overlapped \\
\hline 5. & M.C. & Female & Caucasian & Periapical & First molar & Right & $\begin{array}{l}\text { Entomolaris, } \\
\text { Paramolaris }\end{array}$ & Slightly overlapped \\
\hline 6. & E. D. & Female & Caucasian & Periapical & $\begin{array}{l}\text { First molar, } \\
\text { Second molar }\end{array}$ & Left & Entomolaris & Moderately overlapped \\
\hline 7. & P.K. & Female & Caucasian & Periapical & First molar & Left & Entomolaris & Slightly overlapped \\
\hline 8. & K.M. & Female & Caucasian & Periapical & Second molar & Right & Entomolaris & Slightly overlapped \\
\hline 9. & B.P. & Female & Caucasian & Periapical & First molar & Right & Entomolaris & Moderately overlapped \\
\hline 11. & N.N. & Female & Caucasian & Periapical & Second molar & Left & Entomolaris & Moderately overlapped \\
\hline 12. & K.G. & Female & Caucasian & Panoramic & First molar & Bilateral & Entomolaris & Slightly overlapped \\
\hline 13. & N.N. & Male & Asian & Panoramic & First molar & Bilateral & Entomolaris & $\begin{array}{l}\text { Right — slightly overlapped; } \\
\text { Left — severely overlapped }\end{array}$ \\
\hline
\end{tabular}

In the course of investigating RE and RP the researchers developed a number of different classifications.

Calrsen and Alexandersen [7] elaborated a classification based on the localisation of the cervical part of $R E$, according to which:

- Type A: RE is located lingually to the distal root complex which has 2 cone-shaped macrostructures;

- Type B: RE is located lingually to the distal root complex which has 1 cone-shaped macrostructure;

- Type C: RE is located lingually to the mesial root complex;

- Type AC: RE is located lingually between the mesial and distal root complexes.

There are two alternative classifications of RE, one proposed by Ribeiro and Consolaro (1997) [22] describing the curvature of the root or the root canal, and second presented in 2010 by Song et al. [25] based on computed tomography (CT).

Only one classification of RP can be found in the literature of the subject. It was proposed by Calrsen and Alexandersen [7] who divided RP into two types [12]:

- Type A: cervical part is located on the mesial root complex;

- Type B: cervical part is located centrally between the mesial and distal root complexes.

The aim of the paper is to present radiographic appearances of RE and RP and indicate clinical implications of its presence.

\section{MATERIALS AND METHODS}

The material consisted of the radiographs taken from the database of the Department of Dental and Maxillofacial Radiodiagnostics of the Medical University in Lublin. Intraoral and extraoral radiographs taken between 2011 and 2013 were carefully screened for additional roots in mandibular first and second molars. While searching through the database for RE and PR cases, the main issue was to determine whether in a radiograph one could differentiate between a separate additional root or an hourglass-shaped single root with double periodontum.

\section{RESULTS}

Among 3,000 panoramic radiographs and 300 periapical radiographs of first and second mandibular molars screened for RE and RP in the Department of Dental and Maxillofacial Radiodiagnostics of the Medical University in Lublin, only 14 cases were found; 11 teeth in the Polish population and 3 in Taiwanese population. Only 2 panoramic radiographs revealed the presence of RE and, surprisingly, the occurrence was bilateral. Ten cases of RE were found in the first molar and 3 cases in the second molar. In 1 radiograph, RE was found both in the first and the second molar. Unexpectedly, one first molar had 4 roots, which means that it had both RE and RP. Six teeth were found on the right side of the mandible and 8 on the left side. The results are presented in detail in Table 1 (Figs. 1-4). 


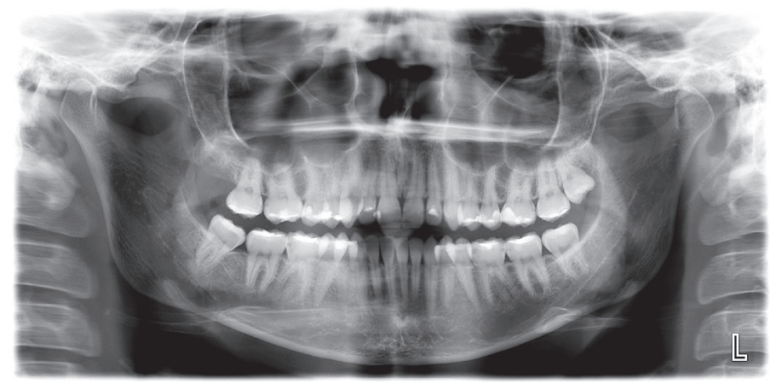

Figure 1. Extraoral panoramic examination - bilateral occurrence of radix entomolaris in first mandibular molars.

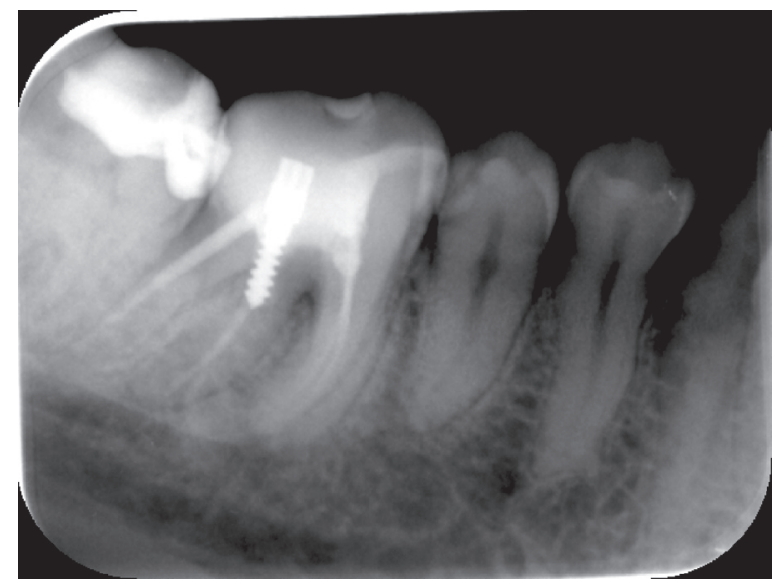

Figure 2. Intraoral periapical radiograph; tooth 46 — radix entomolaris and paramolaris.

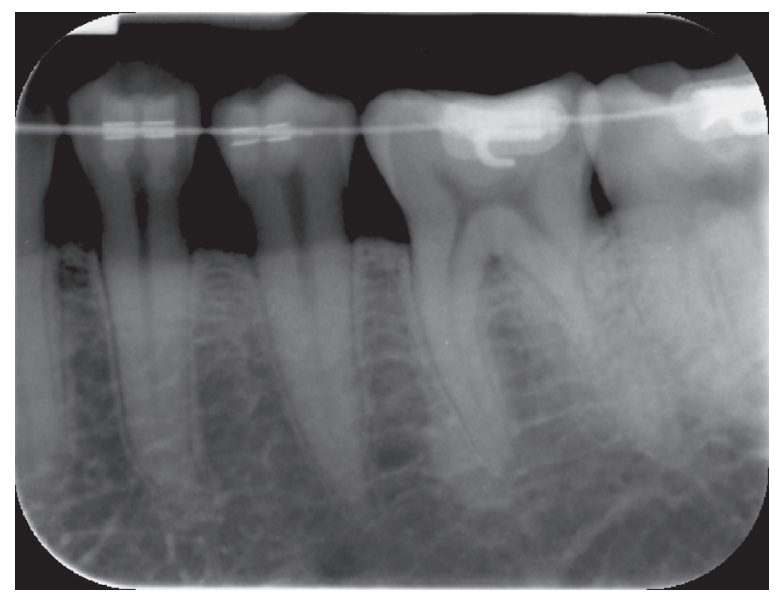

Figure 3. Intraoral periapical radiograph; tooth 36 - radix entomolaris.

\section{DISCUSSION}

A lot of studies have been conducted over the last few years on RE and RP, many of which tried to expose and account for the connection between the occurrence of RE/RP and the subjects' race, sex, age,

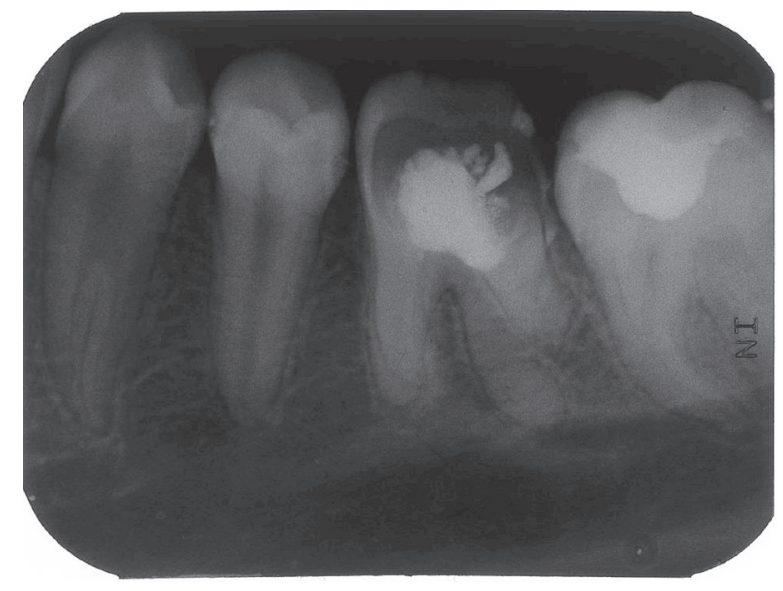

Figure 4. Intraoral periapical radiograph; tooth 36 — radix entomolaris.

genetic traits, as well as its location (unilateration and bilateration). Most studies focused only on first molars $[2,3,9,28,30]$, because it was noticed that the occurrence of RE was the highest there. However, some studies took into consideration also the third molars [21], or concentrated on all mandibular molars [26].

In all the reviewed papers, RE was found in either of mandibular molars with the second molar revealing always the lowest RE prevalence [27, 29]. Interestingly, there are some studies which report the occurrence of RE in primary teeth $[14,15,20]$.

Dental practitioners search for an additional root with the use of different radiological methods: intraoral periapical radiographs, spiral $\mathrm{CT}$ and cone beam computed tomography (CBCT).

Most of epidemiological studies were conducted with the use of intraoral periapical radiographs. They are focused on the prevalence of RE in different populations (Curzon [10], De Moor et al. [12], Ferraz and Pecora [13], Suyambukesan and Perumal [26]).

In many research studies an attempt was made to explain the aetiology of RE appearance but no conclusive answers could be given. Additional root formation can be related to external factors during odontogenesis, or penetration of an atavistic gene, which reappears after several generations of absence. In eumorphic roots, racial genetic factors influence the more profound expression of a particular gene that results in the more pronounced phenotypic manifestation [22, 23].

Only Curzon (1974) [10] suggested more specifically that the trait of RE appearance had a high degree 
of the genetic penetrance. He connected the fact with the similar prevalence of RE in the pure Eskimo population and Eskimo/Caucasian mixes.

He was also convinced that RE occurrence should be attributed to a genetic rather than developmental variation. In addition, he suggested that the feature responsible for $\mathrm{RE}$ occurrence is dominant.

Upon evaluating the feature of unilateral or bilateral occurrence of RE, Suyambukesan and Perumal [26] showed no bilateral occurrence of RE. Contrary to Suyambukesan and Perumal [26] there are some studies which proved the bilateral occurrence of RE in first mandibular molars ranging from $50 \%$ to $67 \%$ of the cases (Yew 1993 and Steelman 1986).

De Moor et al. [12] investigated the occurrence of RE depending on the gender and the side of its occurrence. They revealed that there was no significant difference in RE occurrence depending on the gender and there was more frequent occurrence of RE on the left side of the mandible than on the right.

By contrast, there are much fewer studies on the prevalence of RP. In the study by Visser [see 11] the following occurrence rate of RP was observed: $0 \%$ of $\mathrm{RP}$ in the first mandibular molar, $0.5 \%$ in the second mandibular molar and $2 \%$ in the third mandibular molar. The conclusion is that RP occurs at a much lower frequency than RE.

Wang et al. [30] studied the correlation between the morphology of RE and its radiological image and estimated the best horizontal angulation for RE detection. On examining the Chinese population using intraoral periapical radiographs in 7 different horizontal angles they determined 3 types of resulting radiological image of $\mathrm{RE}$ :

- Slightly overlapped image of RE, i.e. one in which the RE is identifiable most easily;

- Moderately overlapped image of RE;

- Severely overlapped image of RE.

The authors searched for the best angle in visualising the RE depending on its type. They concluded that mesial projections are better than distal in detecting extra roots. The best angle to capture RE was 25 degree in mesial projection [30].

To sum up, 2-dimensional radiographs are useful in searching for and recognising additional roots. Below we present some guidelines helpful during radiographs interpretation. In recognising the additional root several characteristic radiological features can be observed:
- Crossing of the translucent line in the pulp space;

- Crossing of the periodontal ligament space;

- Unclear view or outline of the distal root contour or the root canal;

- Obvious visualisation of the extra root between the mesial and distal roots [26].

\section{Study based on spiral CT}

Kumar et al. [16] presented a case report of the patient with bilateral RE occurrence in the first mandibular molars. They proved that spiral CT was helpful in detecting extra roots, especially on axial scans. The recommendation for this examination is limited due to some disadvantages such as a high dose of radiation, high cost, moderate availability, poor resolution and difficulty in interpretation.

\section{Studies based on CBCT}

In order to detect $\mathrm{RE}$ and $\mathrm{PR}$, more and more studies rely on CBCT. This examination is valuable, especially in detecting the curvature of the root.

Abella et al. [1] focused on the knowledge of the RE curvature emphasizing the prevention of endodontic files separation during treatment.

CBCT (axial slices) were also used by Tu et al. [28] to study first mandibular molars in the Chinese population. Apart from the epidemiological implications of the study, the most important finding was that the axial plane of CBCT allows for the exact visualisation of the distolingual orifice in relation to other canals.

The authors argued that CBCT examination was not suitable for examining the root canals which are narrower than $0.25 \mathrm{~mm}$. For this purpose micro-CT provides a better imaging modality, but it can only be applied in in vitro studies [28].

\section{Clinical approach}

Apart from radiological examination which is crucial in detecting additional roots, the clinical examination can also play a significant role. An extra cusp (tuberculum paramolare) or a more prominent occlusal distal or distolingual lobe, in combination with a cervical prominence or convexity, can indicate the presence of an additional root [6]. Kim et al. [15] claim that there is a correlation between the crown appearance and the presence of RE. The study by Kim et al. [15] was conducted on the first permanent and primary second molars. It reported significantly larger intercuspal distances between the distobuccal-distolingual cusp tips and a larger distal-area buccolingual width than those without RE. Remarkably, there was 
no significant correlation between the existence of a sixth cusp and the presence of RE.

The knowledge of an additional root is essential not only in endodontics but in other fields of dentistry such as surgery, orthodontics and periodontics.

\section{Implications for endodontics}

A significant number of case reports focus on possible problematic endodontic treatment involving teeth with RE or RP occurrence. The majority of those describe endodontic treatment of the first mandibular molars with RE/RP [4-6, 11, 16-18], one was found describing the presence of RE in the second molar [8] and 2 studies reporting RE in the third molar $[21,26]$.

In endodontic therapy the cavity opening should be modified in distolingual direction from a triangular to trapezoid shape [12].

According to Vivekananda and Vinod [29] the orifice of RE is located disto- to mesio-lingualy to main canal or canals in the distal root whereas the orifice of RP is located mesiobuccally to the distal orifice. This information can be helpful in searching for an additional canal. The knowledge of the curvature types of RE helps avoid instrument separation. This feature can be evaluating only based on 3-dimentional examinations. The study of Yu et al. [31] revealed that there were more instrument separations, lateral perforations and underfilled root canals among all first mandibular molars with RE treated endodontically than in other canals of mandibular first molars. Another iatrogenic error was mentioned in Mirikar's et al. case report [19]. They described the procedure of non-surgical treatment of furcal perforation of the tooth with RE.

Long-term unhealed periapical lesions can be caused by the fact that the root canal in RE/RP was not found and, therefore, not filled.

\section{Implications for periodontics}

The connection between RE and the destruction of periodontal tissues is rare but Sachdeva and Phadnaik [24] presented a case report of a young girl with bilateral RE occurrence in the mandibular first molar. It contributed to the moderate destruction of the bone with involvement of the distally localised furcation.

\section{Implications for prosthetics}

When dealing with the missing teeth, to restore the space one can plan a prosthetic bridge. When a tooth with RE/PR did not receive a proper endodontic treatment due to instrument separation, perforation, unfilled root canal or unhealed periapical lesion, the consequence is that such a tooth is not suitable for any prosthetic restoration such as bridge or post and core with crown.

\section{Untreated RE/RP as a focal infection}

Patients with malignant tumours are referred to the dental practitioners in order to have possible foci of infection of odontogenic origin eliminated before bone marrow transplant or chemotherapy. This includes RE/RP.

Failure to find and fill a root canal in an additional root can promote the process of inflammation which can be dangerous for the life of such immunocompromised patients. By knowing this variant of anatomy and careful interpreting of radiological examination dentists can save patients' life.

\section{CONCLUSIONS}

The knowledge of the RE and RP occurrence is crucial because it affects the results of the treatment in all fields of dentistry, especially in endodontics. When one suspects the RE occurrence, a careful radiograph interpretation is required. To confirm the suspicion an additional intraoral radiograph in the oblique projection should be taken - the best visibility is obtained in 25 degree, in mesial projection. CBCT examination can also confirm the occurrence of RE or RP. This particular examination is helpful in evaluating the type of root curvature. The occurrence rate of RE and RP in the Caucasian population is lower than in other populations, however dentists should be aware of the possibility of such an occurrence. Dental practitioners should know characteristic features of RE and RP and be familiar with recognising them with the use of various clinical and radiological methods.

\section{REFERENCES}

1. Abella F, Mercadé M, Duran-Sindreu F, Roig M (2011) Managing severe curvature of radix entomolaris: three-dimensional analysis with cone beam computed tomography. Int Endod J, 44: 876-885.

2. Attam K, Nawal RR, Utneja S, Talwar S (2012) Radix Entomolaris in mandibular first molars in Indian population: a review and case reports. Case Rep Dent, 2012; 2012: 595494. doi: 10.1155/2012/595494 (Epub 2012 Oct 22)

3. Bharti R, Arya D, Saumyendra VS, Kulwinder KW, Tikku AP, Chandra A (2011) Prevalence of radix entomolaris in an Indian population. Indian J Stomatol, 2: 165-167.

4. Bolla N, Naik BD, Kavuri SR, Sriram SK (2013) Radix entomolaris: a case report. Endodontology, 25: 121-124. 
5. Boruah L, Nautiyal G, Arunagiri D (2010) Radix paramolaris and entomolaris - report of three cases in an Indian dental school. Endo, 4: 91-95.

6. Calberson FL, De Moor RJ, Deroose CA (2007) The radix entomolaris and paramolaris: clinical approach in endodontics. J Endod, 33: 58-63.

7. Carlsen O, Alexandersen V (1990) Radix entomolaris: identification and morphology. Scand J Dent Res; 98: 363-373.

8. Chandra SS, Chandra S, Hussain N, Ahuja A (2012) Endodontic therapy in a 3-rooted mandibular second molar: a case report. IDJSR, 1: 23-28.

9. Chandra SS, Chandra S, Shankar P, Indira R (2011) Prevalence of radix entomolaris in mandibular permanent first molars: a study in a South Indian population. Oral Surg Oral Med Oral Pathol Oral Radiol Endod, 112: 77-82.

10. Curzon MEJ (1974) Miscegenation and the prevalence of three-rooted mandibular first molars in Baffin Eskimo. Comm Dent Oral Epidemiol, 2: 130-131.

11. Davini $F$, Cunha RS, Fontana $C E$, de Magalhães Silveira $C F$, da Silveira Bueno CE (2012) Radix entomolaris: a case report. RSBO, 9: 340-344.

12. De Moor RJ, Deroose CA, Calberson FL (2004) The radix entomolaris in mandibular first molars: an endodontic challenge. Int Endod J, 37: 789-799.

13. Ferraz JAB, Pécora JD (1992) Three-rooted mandibular molars in patients of Mongolian, Caucasian and Negro origin. Braz Dent J, 3: 113-117

14. Gupta N, Goswami M, Singh K (2013) Bilateral radix entomolaris with primary and permanent mandibular first molars. BMJ Case Rep, 2013; doi: 10.1136/bcr-2013200018 (Epub 2013 Jul 12).

15. Kim KR, Song JS, Kim SO, Kim SH, Park W, Son HK (2013) Morphological changes in the crown of mandibular molars with an additional distolingual root. Arch Oral Biol, 58: 248-253.

16. Kumar R, Patil S, Hoshing U, Medha A, Mahaparle R (2011) Bilateral mandibular first molars with anatomical variations confirmed with spiral computerised tomography. Endo, 5: 125-131.

17. Madhuram K, Keerthana S, Rajkumar S, Sankari SL (2011) Radix entomolaris: report of two cases. IJMD, 1: 227-230.

18. Mahendra M, Verma A, Tyagi A, Singh S, Chsddha R, Malviya $K$ (2013) Management of complex root canal curvature of bilateral radix entomolaris: three dimensional analysis with cone beam computed tomography. Case Rep Dent, 2013; doi: 10.1155/2013/697323 (Epub 2013 Nov 13).

19. Mirikar P, Shenoy A, Mallikarjun GK (2009) Nonsurgical management of endodontic mishaps in a case of radix entomolaris. J Conserv Dent, 12: 169-174.

20. Nagaveni NB, Umashankara KV (2012) Radix entomolaris and paramolaris in children: a review of the literature. J Indian Soc Pedod Prev Dent, 30: 94-102.

21. Park JB, Kim N, Park S, Kim Y, Ko Y (2013) Evaluation of number of roots and root anatomy of permanent mandibular third molars in a Korean population, using conebeam computed tomography. EJD, 7: 296-301.

22. Rebeiro FC, Consolaro A (1997) Importancia clinica y antropologica de la raiz distolingual en los molars inferiors permanents. Endodoncia, 15: 72-78.

23. Reichart PA, Metah D (1981) Three-rooted permanent mandibular first molars in the Thai. Comm Dent Oral Epidemiol, 9: 191-192.

24. Sachdeva S, Phadnaik MB (2012) Three-rooted mandibular first molar: a consideration in periodontal therapy. J Indian Soc Periodontol, 16; 286-289.

25. Song JS, Choi HJ, Jung IY, Jung HS, Kim SO (2010) The prevalence and morphologic classification of distolingual roots in the mandibular molars in a Korean population. JOE, 36: 653-657.

26. Suyambukesan S, Perumal GL (2013) Radiographic detection of additional root on mandibular molars in Malaysian population a prevalence study. JRID, 3: 31-38.

27. Thoden Van Veizen SK, Wesselink PR, De Cleen MJH. Endodontology (1995) $2^{\text {nd }}$ Ed. Boha Stafleu Van Loghum, Houtem/Diegem, pp.142-143.

28. Tu MG, Huang HL, Hsue SS, Hsu JT, Chen SY, Jou MJ, Tsai CC (2009) Detection of permanent three-rooted mandibular first molars by Cone-Beam Computed Tomography imaging in Taiwanese individuals. JOE, 35: 503-507.

29. Vivekananda RK, Vinod BM (2010) Clinical management of radix entomolaris and radix paramolaris: case reports. AEDJ, 2: 99-102.

30. Wang Q, Yu G, Zhou XD, Peters OA, Zheng QH, Huang DM (2011) Evaluation of x-ray projection angulation for successful radix entomolaris diagnosis in mandibular first molars in vitro. JOE, 37: 1063-1068.

31. Yu G, Ye L, Huang D (2012) Clinical investigation of radix entomolaris in mandibular first molars. WCJS, 30; 259-261. 